\title{
Psychosis in Alzheimer disease - mechanisms, genetics and therapeutic opportunities
}

\author{
Zahinoor Ismail $\mathbb{1}^{1,7}$, Byron Creese $\mathbb{1}^{2,7}$, Dag Aarsland ${ }^{3}$, Helen C. Kales ${ }^{4}$, \\ Constantine G. Lyketsos ${ }^{5}$, Robert A. Sweet $\mathbb{B}^{6}$ and Clive Ballard $\mathbb{B}^{3 凶}$
}

Abstract | Psychosis is a common and distressing symptom in people with Alzheimer disease, and few safe and effective treatments are available. However, new approaches to symptom assessment and treatment are beginning to drive the field forward. New nosological perspectives have been provided by incorporating the emergence of psychotic symptoms in older adults even in advance of dementia - into epidemiological and neurobiological frameworks as well as into diagnostic and research criteria such as the International Psychogeriatric Association criteria for psychosis in neurocognitive disorders, the Alzheimer's Association International Society to Advance Alzheimer's Research and Treatment (ISTAART) research criteria for psychosis in neurodegenerative disease, and the ISTAART criteria for mild behavioural impairment. Here, we highlight the latest findings in genomics, neuroimaging and neurobiology that are informing approaches to drug discovery and repurposing. Current pharmacological and non-pharmacological treatment options are discussed, with a focus on safety and precision medicine. We also explore trial data for pimavanserin, a novel agent that shows promise for the treatment of psychosis in people with dementia, and discuss existing agents that might be useful but need further exploration such as escitalopram, lithium, cholinesterase inhibitors and vitamin D. Although the assessment and management of psychosis in people with dementia remain challenging, new opportunities are providing direction and hope to the field.

Neuropsychiatric symptoms (NPS) are almost ubiquitous in people with dementia, with prevalence estimates as high as $97 \%{ }^{1}$. These non-cognitive symptoms are distressing not only for people with Alzheimer disease (AD) or other dementias but also for their caregivers, and are associated with poor outcomes in terms of function, quality of life, disease course, mortality and economic $\cos \mathrm{t}^{2,3}$. Also known as behavioural and psychological symptoms of dementia (BPSD), these NPS include impairments in motivation, interest, social behaviour and awareness, mood disorders, anxiety, agitation, impulsivity, and hallucinations and delusions, all of which often require clinical intervention. However, reliable and safe treatments for NPS have remained elusive $e^{4-6}$.

Psychotic symptoms, which consist of hallucinations and delusions, are among the most clinically relevant NPS, and are associated with hospitalization or institutionalization, cognitive and functional impairment, accelerated cognitive decline and mortality, as well as caregiver distress $^{7-12}$. A systematic review reported psychosis in $41 \%$ of people with $\mathrm{AD}^{13}$. In this sample, $23 \%$ of participants had delusions only, 5\% had hallucinations only, and $13 \%$ had both delusions and hallucinations.

In this Review, we describe the main features of psychosis in AD and other dementias and their associations with disease stage and severity. We also consider how recent insights into the mechanisms underlying $\mathrm{AD}$-related psychosis are informing new treatment approaches.

\section{Psychosis and cognitive decline Links to disease stage and severity}

Delusions and hallucinations in AD are associated with different patient characteristics: in a memory clinic sample of people with probable $\mathrm{AD}$, delusions were associated with older age, depression and aggression, whereas hallucinations were associated with more severe dementia and longer duration of illness ${ }^{14}$. An analysis of National Alzheimer's Coordinating Center (NACC) data found that delusions and hallucinations also showed differential associations with cognition and function, with hallucinations conferring greater cognitive and 


\section{Key points \\ - Psychosis is surprisingly common in Alzheimer disease (AD) and can emerge as part of the neurodegenerative disease process in advance of dementia during the mild cognitive impairment stage or even earlier. \\ - Subtypes of psychotic symptoms - that is, persecutory versus misidentification delusions or delusions versus hallucinations - have different trajectories and neurobiological correlates. \\ - Both schizophrenia and $\mathrm{AD}$ risk genes might be involved in $\mathrm{AD}$ psychosis. \\ - Tau tangle burden and kalirin-mediated synaptic dysfunction seem to be neurobiological components of psychosis in AD. \\ - Of the currently available antipsychotic drugs, aripiprazole seems to provide the best balance between efficacy and safety in people with AD psychosis; pimavanserin has shown promise in Parkinson disease-related psychosis and data from AD trials are compelling. \\ - A better understanding of the natural history and neurobiological underpinnings of psychosis in neurodegenerative disease will drive drug development and repurposing of existing drugs.}

functional deficits than delusions ${ }^{15}$. Individuals with delusions might represent a heterogenous group: persecutory delusions, such as delusions of theft, are the most common late-life delusions ${ }^{16}$ and occur earlier in the AD course than do misidentification delusions, such as TV sign and phantom boarder (see below), which are associated with advanced dementia and greater cognitive impairment ${ }^{17}$.

The relative prevalence of psychotic symptoms varies according to the underlying dementia diagnosis. Among non-AD dementias, psychosis occurs most frequently in dementia with Lewy bodies (DLB; 75\% prevalence), followed by Parkinson disease (PD) dementia (50\%), vascular dementia (15\%) and frontotemporal dementia $(10 \%)^{18}$. Longitudinal data indicate that, in both $\mathrm{AD}$ and DLB, NPS are often present at the time of diagnosis ${ }^{19}$ but psychosis is more prevalent, persistent and intense in DLB and $\mathrm{PD}^{20}$. The presence of psychosis, combined with clinicians' preconceived notions about psychosis and dementia, can influence a dementia diagnosis. In a study of 961 neuropathologically confirmed cases of $\mathrm{AD}$ in the NACC database, the presence of psychosis in individuals with $\mathrm{AD}$ was associated with a fivefold increased likelihood of misdiagnosis of the condition as $\mathrm{DLB}^{21}$, suggesting that clinicians tend to favour a DLB diagnosis in the presence of psychosis. In a related study in neuropathologically confirmed AD cases, psychosis was

\footnotetext{
Author addresses

${ }^{1}$ Departments of Psychiatry, Clinical Neurosciences, Community Health Sciences, and Pathology, Hotchkiss Brain Institute and O’Brien Institute of Public Health, University of Calgary, Calgary, AB, Canada.

${ }^{2}$ University of Exeter Medical School, College of Medicine and Health, University of Exeter, Exeter, UK.

${ }^{3}$ Department of Old Age Psychiatry, Institute of Psychiatry, Psychology and Neuroscience, King's College London, London, UK.

${ }^{4}$ Department of Psychiatry and Behavioral Sciences, UC Davis School of Medicine, University of California, Sacramento, CA, USA.

${ }^{5}$ Department of Psychiatry and Behavioral Science, Johns Hopkins Bayview, Johns Hopkins University, Baltimore, MD, USA.

${ }^{6}$ Department of Psychiatry, University of Pittsburgh School of Medicine, Pittsburgh,

PA, USA.

'These authors contributed equally: Zahinoor Ismail, Byron Creese.
}

associated with Lewy bodies rather than $\mathrm{AD}$ pathology ${ }^{22}$, suggesting a degree of overlap between amyloidopathies and synucleinopathies that could contribute to diagnostic confusion.

\section{Diagnosis}

Psychotic symptoms are challenging to diagnose in the context of cognitive impairment. In addition to delusions of theft, delusions of infidelity or abandonment, beliefs that deceased individuals are alive, general suspiciousness not related to theft (for example, being plotted against, sent to jail or evicted), and elaborate systematized delusions, including erotomania or religiosity, have been observed in people with $\mathrm{AD}^{23}$. Distinguishing between delusions and forgetfulness or confabulation requires an appreciation of whether information was encoded into memory ${ }^{24}$ : persistence of the belief suggests a delusion, whereas inconsistent statements are more suggestive of confabulations. Even more challenging are misidentification syndromes, which broach both cognitive capacity and perceptual abilities. Beliefs that strangers are residing in one's home (phantom boarder syndrome), loved ones have been replaced by impostors, images on television are real (TV sign) and, in particular, the belief that the person in the mirror is someone else might suggest advanced cognitive impairment ${ }^{25}$, prosopagnosia ${ }^{26}$, impaired self-monitoring ${ }^{27}$, or disruptions in signal processing and/or sensory integration circuitry ${ }^{28}$.

Compared with delusions, hallucinations can be fleeting or more fragmented ${ }^{23}$. Hallucinations can manifest in any sensory modality; however, visual and auditory hallucinations are the most common. Auditory hallucinations can range from noises to full conversations. Visual hallucinations most commonly involve people or animals but can also include faces or deceased individuals, colours, inanimate objects or unformed images ${ }^{29}$. Again, given the overlap between hallucinations and misidentification syndromes, distinguishing between them is not always possible.

In advance of diagnosing psychosis in an older adult, irrespective of cognitive status, reversible and contributing causes must be addressed (see treatment section below). The best-known criteria for psychosis in $\mathrm{AD}$ were published in 2000 by Jeste and Finkel, who described delusions and hallucinations (auditory or visual) in the context of clinically diagnosed $\mathrm{AD}^{30}$. Most measurement instruments for psychosis in $\mathrm{AD}$ have taken a symptomatic - as opposed to syndromic approach. Studies have generally demonstrated reasonable agreement between different instruments, with some subtle differences in the ability to detect symptoms ${ }^{31}$ and change in response to treatment ${ }^{32}$.

Substantial evidence suggests that psychosis can emerge during the prodromal or mild cognitive impairment (MCI) phase of the neurodegenerative disease continuum $^{33}$, and these psychotic symptoms would not be included as part of the disease according to the original Jeste and Finkel criteria. To address this issue, revised criteria have been formulated. The new International Psychogeriatric Association (IPA) criteria for psychosis in major and mild neurocognitive disorders now include $\mathrm{MCI}$ as well as other dementia aetiologies, representing a 
move towards a syndromic understanding of psychosis in dementia ${ }^{34}$. However, other important diagnostic issues, including comorbidity with other NPS syndromes, the risk of dementia if NPS emerge in advance of cognitive impairment, and the role of biomarkers in assessment, diagnosis and work-up, require further attention.

\section{Comorbidities}

Psychosis can co-present with agitation ${ }^{35}$, and the relationship between these two symptoms is sometimes difficult to disentangle, although the treatment approaches are similar. Importantly, agitation was redefined with the publication, in 2015, of the IPA criteria for agitation in cognitive disorders $^{36}$. The importance of these syndromic IPA criteria lies in the description of three domains of agitation (verbal aggression, physical aggression and excessive motor activity), which might be used to improve case definitions and to define different treatment targets. To our knowledge, little evidence is available on the association between psychotic symptoms and the new agitation domains, although preliminary work has explored IPA agitation-specific measurement in $\mathrm{AD}^{37}$.

Affective symptoms can also co-occur with psychotic symptoms, although factor analyses in dementia and MCI have consistently demonstrated that psychotic and affective symptoms load onto separate factors, that is, they associate with different latent variables potentially representing different subsyndromes ${ }^{38,39}$. Nonetheless, when they co-occur, psychotic and affective symptoms in MCI are associated with a higher risk of incident dementia than either factor alone ${ }^{40}$. Importantly, in the new IPA psychosis criteria $^{34}$, agitation and depression can be coded as associated features of syndromic psychosis in neurocognitive disorders, enabling further exploration of NPS comorbidity using these criteria.

\section{Emergence of psychosis in later life}

An important and ongoing issue is the relationship between the emergence of psychotic symptoms in later life and the risk of incident cognitive decline and dementia. As reflected in the Jeste and Finkel criteria for psychosis in $\mathrm{AD}^{30}$, psychotic symptoms were included only when they emerged after a dementia diagnosis. However, psychotic symptoms have been observed in people with MCI. Specifically, hallucinations are present in $1.3-2.6 \%$ and delusions in $3.1-10.5 \%$ of patients with $\mathrm{MCI}^{41}$. Most ${ }^{40,42-44}$ but not all ${ }^{45,46}$ longitudinal studies have found an increased risk of dementia associated with psychosis. For the negative studies, low baseline psychosis frequency in the study sample ${ }^{46}$ and short median duration of follow-up ( 1.58 years $)^{45}$ might have been confounding factors. Although more research is required, the evidence to date suggests that psychosis in $\mathrm{MCI}$ is part of the neurodegenerative disease process, supporting the inclusion of MCI or mild neurocognitive disorders in the new IPA criteria.

Psychotic symptoms that emerge in older adults with ostensibly normal cognition are even more challenging from a nosological perspective. The field currently captures these symptoms under the umbrella terms of delusional disorder, late-onset schizophrenia or very-late-onset schizophrenia-like psychosis $(\text { VLOSLP })^{47}$, and they are usually diagnosed when these older adults present to clinical care. Indeed, a recent descriptive study of late-life delusional disorder highlighted this clinical nomenclature ${ }^{48}$. In a review of 55 cases of delusional disorder in adults aged $\geq 65$ years who presented to a clinical service over a 12 -year period, sustained recovery occurred in $20 \%$ of the individuals and an additional $30 \%$ showed improvement over a mean follow-up period of 38 months $^{49}$. Atypical antipsychotics were prescribed in almost $60 \%$ of cases and, of those who showed improvement, $96 \%$ received these drugs. However, owing to study heterogeneity and/or insufficient follow-up time to meaningfully determine risk, whether these constructs of late-life psychosis are associated with an increased risk of incident cognitive decline and dementia remains unsettled ${ }^{33}$.

Studies that used longer follow-up durations ${ }^{50,51}$, assessed incident psychosis specifically ${ }^{52}$, framed psychosis as an NPS in cognitively normal older adults ${ }^{53}$ or used health databases ${ }^{54-57}$ found associations between late-life psychosis and incident dementia. Interestingly, in a prospective population-based cohort of 23,999 men aged 65-85 years, psychosis was associated with a substantial risk of dementia, most notably in individuals with a shorter duration of psychosis ${ }^{56}$. This finding was replicated in a Swedish population-based cohort study of 15,409 older adults with VLOSLP and 154,090 age-matched controls ${ }^{57}$. The risk of dementia was increased in the individuals with VLOSLP and was highest in the first year after VLOSLP diagnosis.

Neurobiological data are also emerging to link late-life psychosis to neurodegeneration. For example, a post-mortem study has shown an association between argyrophilic grain disease - a four-repeat tauopathy and psychosis in individuals aged $\geq 65$ years $^{58}$. In this age group, people with psychosis had sixfold increased odds of having this neurodegenerative condition compared with non-psychiatric controls.

As the evidence base evolves, the findings increasingly indicate that late-life psychosis (captured in the clinic as delusions, late-onset schizophrenia, VLOSLP, or NPS) can represent non-cognitive symptoms of neurodegenerative disease and might manifest in advance of cognitive impairment in some individuals. The improved detection of individuals who present with psychosis as the index manifestation of neurodegeneration could potentially offer a window for the prevention of dementia.

\section{Nosology}

To guide and standardize nosology, support phenotypic classification of psychotic symptoms, foster research into psychosis in $\mathrm{AD}$ and related dementias, and pave the way for the systematic implementation of biomarkers for psychosis in $\mathrm{AD}$, the Alzheimer's Association International Society to Advance Alzheimer's Research and Treatment (ISTAART) recently developed research criteria entitled Towards Better Phenotypic Classification and Biomarker Research in $\mathrm{AD}$ and Related Dementias ${ }^{59}$. These criteria, which were co-developed with the IPA psychosis clinical criteria $^{34}$, aim to improve epidemiological, biomarker and genetics research in the field. Research utilizing 
these criteria is expected to provide new insights into the relationship between late-life emergent psychotic symptoms across the cognitive continuum and incident cognitive decline and dementia, focusing on neurobiology and disease mechanisms $s^{59}$. This research could inform drug development to reduce psychotic symptom burden in older adults with cognitive impairment and to prevent or delay the incident dementia associated with late-life psychosis.

\section{Mechanistic insights \\ Genomics}

In recent years, we have seen important advances in identifying the genomic correlates of psychosis in $\mathrm{AD}$ (TABLE 1). Early familial studies estimated the heritability of $\mathrm{AD}$ psychosis at $61 \%^{60}$. A major international genome-wide association study (GWAS) in $>12,000$ individuals with $\mathrm{AD}$, which was published in 2021, was the first to use single nucleotide polymorphisms (SNPs) to calculate the heritability of psychosis in $\mathrm{AD}^{61}$. The heritability estimates from this study ranged from $18 \%$ to $31 \%$ depending on the method used, which is broadly similar to major depressive disorder and schizophrenia. This study also reported the first genome-wide significant risk loci for psychosis in $\mathrm{AD}$, which were located in ENPP6 and SUMF1. ENPP6 is expressed in oligodendrocytes, and the transcript containing the SUMF1 SNPs is found in the cerebral cortex. However, further investigation is required to establish whether these genes are mechanistically linked to psychosis.

Table 1 | Genomic correlates of psychosis in AD

\begin{tabular}{|c|c|}
\hline Gene or disease & Summary of findings \\
\hline \multicolumn{2}{|l|}{ Single genes } \\
\hline ENPP6 (REF. $\left.{ }^{61}\right)$ & rs9994623 identified by GWAS (OR 1.16, 95\% CI 1.10-1.22, $P=1.26 \times 10^{-8}$ ) \\
\hline SUMF1 (REF. ${ }^{61}$ ) & rs201109606 identified by GWAS (OR 0.65, 95\% Cl 0.56-0.76, $P=3.24 \times 10^{-8}$ ) \\
\hline APOE $\varepsilon 4$ allele ${ }^{61,62}$ & $\begin{array}{l}\text { Latest GWAS ( } n>12,000) \text { reported a small but statistically significant association with } \\
\text { psychosis in AD (OR 1.21); findings were inconsistent in previous studies, possibly owing } \\
\text { partly to small sample sizes }\end{array}$ \\
\hline$G B A^{75}$ & $\begin{array}{l}\text { In Parkinson disease, a meta-analysis showed a twofold increased risk of psychosis in } \\
\text { GBA mutation carriers relative to non-carriers; one small study linked GBA mutations } \\
\text { to increased risk of psychosis in dementia with Lewy bodies }\end{array}$ \\
\hline TBX15 $\left(\right.$ REF $\left.^{74}\right)$ & $\begin{array}{l}\text { Differentially methylated region spanned } 10 \mathrm{CpG} \text { sites }(613 \mathrm{bp}) \text { within the first exon of the } \\
\text { gene; hypomethylation in people with } \mathrm{AD} \text { psychosis was consistent across the prefrontal } \\
\text { cortex, superior temporal gyrus and entorhinal cortex }\end{array}$ \\
\hline WT1 $\left(\right.$ REF. $\left.^{74}\right)$ & $\begin{array}{l}\text { Differentially methylated region spanned } \mathrm{CpG} \text { sites }(476 \mathrm{bp}) \text { in the first intron of the WT1 } \\
\text { gene; hypomethylation in people with AD psychosis was consistent across the prefrontal } \\
\text { cortex, superior temporal gyrus and entorhinal cortex }\end{array}$ \\
\hline \multicolumn{2}{|l|}{ Polygenic risk scores } \\
\hline Schizophrenia ${ }^{61,64,65}$ & $\begin{array}{l}\text { Mixed findings; no association in a study using a PRS comprised of } 101 \text { genome-wide } \\
\left.\text { significant } S N P s \text { for schizophrenia (OR } 0.91,95 \% \mathrm{Cl} 0.84-0.97, P=0.006, R^{2}=0.003\right) \text {; } \\
\text { higher PRS associated with psychosis in } \mathrm{AD} \text { in a study using a PRS at various } S N P \text { inclusion } \\
\text { thresholds; optimum } R^{2}(0.008) \text { at } 8,709 \mathrm{SNPs} \text { with the association driven by individuals } \\
\text { with delusions (OR } 1.18,95 \% \mathrm{Cl} 1.06-1.30, P=0.001) \text {; no clear evidence in a larger analysis } \\
\text { overlapping with both of the above data sets }\end{array}$ \\
\hline Major depression ${ }^{65}$ & No association \\
\hline Bipolar disorder ${ }^{61}$ & $\begin{array}{l}\text { Tested at various SNP inclusion thresholds; optimum } R^{2}\left(3.7 \times 10^{-3}\right) \text { at } 79,818 \text { SNPs } \\
\text { (OR } 0.95,95 \% \mathrm{Cl} 0.91-0.99, P=0.025)\end{array}$ \\
\hline $\mathrm{AD}^{61}$ & $\begin{array}{l}\text { Tested at various SNP inclusion thresholds; optimum } R^{2}\left(2.7 \times 10^{-3}\right) \text { at } 195,758 \text { SNPs } \\
\left.\text { (OR } 1.1,95 \% \mathrm{Cl} 1.05-1.14, P=2.07 \times 10^{-5}\right)\end{array}$ \\
\hline \multicolumn{2}{|l|}{ Genetic correlation $^{61}$} \\
\hline$A D$ & No association \\
\hline Schizophrenia & No association \\
\hline Bipolar disorder & Negative correlation $\left(r_{g}=-0.287, P=0.048\right)$ \\
\hline Depressive symptoms & Positive correlation $\left(r_{g}=0.327, P=0.021\right)$ \\
\hline $\begin{array}{l}\text { Amyotrophic lateral } \\
\text { sclerosis }\end{array}$ & No association \\
\hline Parkinson disease & No association \\
\hline Years of schooling & Negative correlation $\left(r_{\mathrm{g}}=-0.312, P=0.005\right)$ \\
\hline Intelligence & No association \\
\hline
\end{tabular}

AD, Alzheimer disease; GWAS, genome-wide association study; PRS, polygenic risk score (a summary score of an individual's genetic risk for a disease or condition across many genetic variants, with a higher score indicating a greater degree of genetic liability); $R^{2}$, proportion of variance in psychosis in AD explained by PRS; $r_{r}$, genetic correlation (proportion of variance shared between the specific condition in the left-hand column and psychosis in AD); SNP, single nucleotide polymorphism. 


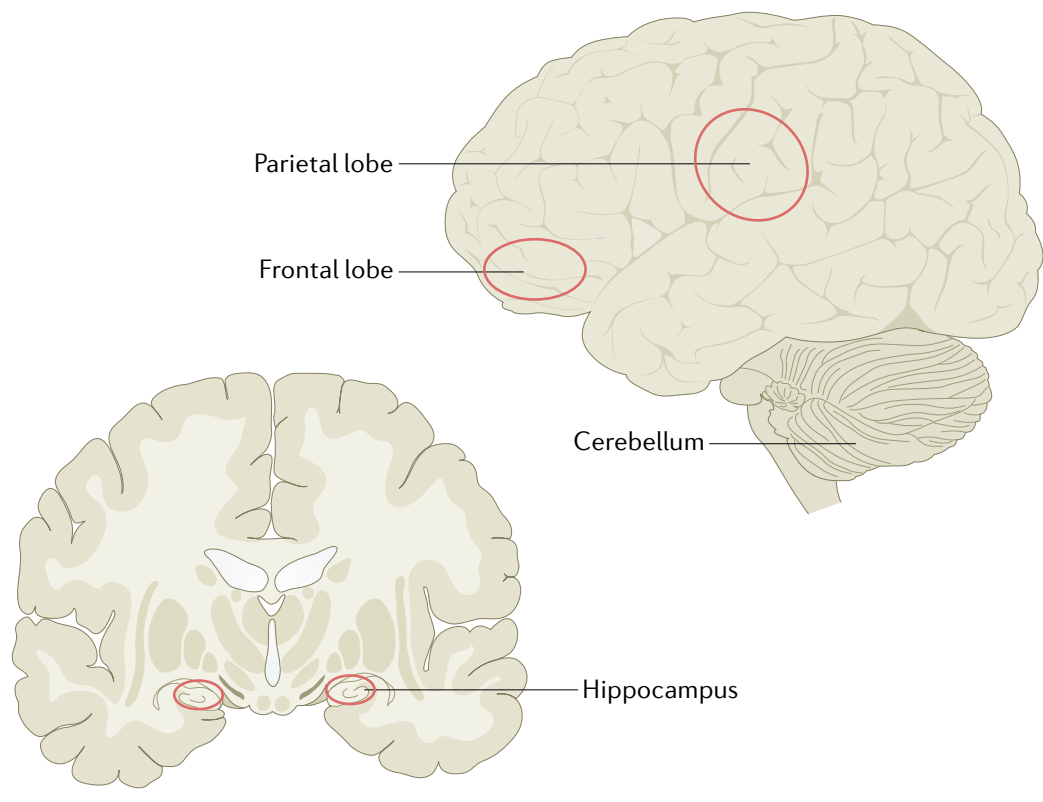

Fig. 1 | Neuroimaging findings in Alzheimer disease psychosis. Neuroimaging findings are diverse, although some relationships with specific psychotic domains have been observed. Delusions have been associated with left frontal atrophy and misidentification delusions have been associated with hippocampal atrophy. Delusions have been associated with default mode network disruption, including parietal and cerebellar atrophy, but no default mode network signal was associated with a composite psychosis score. Hallucinations have been associated with supramarginal atrophy within the parietal lobe.
Psychosis in $\mathrm{AD}$ is a complex trait, and its genetics mirrors that of other psychiatric traits in that common SNPs alone are likely to only explain a small proportion of the variance ${ }^{70}$. Study of the epigenome has led to major advances in the understanding of the molecular mechanisms underlying a variety of psychiatric and neurological conditions $\mathrm{s}^{71-73}$. Hypomethylation in the TBX15 and WT1 genes, which have been implicated in the pathophysiology of $\mathrm{AD}$, was found in the dorsolateral prefrontal cortex, superior temporal gyrus and entorhinal cortex in individuals with $\mathrm{AD}$ psychosis ${ }^{74}$. Interestingly, and reflecting some of the emerging data from genomic studies, the top-ranked differentially methylated regions also showed enrichment for known schizophrenia-associated genetic and epigenetic variants. Genomic research in non-AD dementias remains scarce. Mutations in the glucocerebrosidase (GBA) gene have been associated with psychotic symptoms and progression to dementia in $\mathrm{PD}^{75}$. However, to our knowledge, only one study of GBA in DLB has been published, which described an increased frequency of hallucinations among GBA mutation carriers ${ }^{76}$.

\section{Neuroimaging}

Neuroimaging studies have implicated several regions of the brain in AD psychosis, as summarized in FIC. 1. Early structural and functional imaging studies, most of which examined delusions rather than a combined delusion-hallucination construct, pointed towards increased frontotemporal atrophy and neocortical hypometabolism in people with AD psychosi ${ }^{24,77}$. Later studies supported these associations. An analysis of the NACC cohort revealed accelerated atrophy in frontal and temporal lobes in individuals with delusions ${ }^{78}$, and other studies in independent cohorts have reported links with hippocampa ${ }^{79}$ and parahippocampal ${ }^{80}$ atrophy. The parahippocampal atrophy was implicated in delusional misidentifications, consistent with findings from neuropathological studies ${ }^{81}$. Another study of Alzheimer's Disease Neuroimaging Initiative (ADNI) data found that cortical thinning in the supramarginal area of the parietal lobe was a risk factor for hallucinations ${ }^{82}$. However, the fact that only a small minority of people experience hallucinations without delusions might have been a confounding factor in this study.

In a longitudinal study, again using the ADNI cohort, Fischer et al. ${ }^{83}$ reported an increased rate of grey matter atrophy in the cerebellum and parietal lobe prior to the onset of delusions. The lack of involvement of frontal areas may be surprising but the authors highlight possible links between posterior cortical atrophy and frontal circuits and the default mode network (DMN). The DMN has been the focus of a number of functional imaging studies in recent years because of the wider literature showing that it is disrupted in $\mathrm{AD}$ and other disorders characterized by psychosis. However, two further studies examined a composite construct of psychosis consisting of delusions, hallucinations and nighttime behaviours as rated on the Neuropsychiatric Inventory (NPI) and found no DMN association ${ }^{84,85}$. A third study found that delusions in people with $\mathrm{AD}$ were associated with decreased connectivity within the $\mathrm{DMN}^{86}$. 
White matter changes have been the focus of relatively few studies in $\mathrm{AD}$ and the results have been inconsistent $\mathrm{t}^{24,87}$.

Most studies of regional perfusion and metabolic changes in $\mathrm{AD}$ psychosis, as measured by single-photon emission CT (SPECT) and ${ }^{18} \mathrm{~F}-\mathrm{FDG}$ PET, were conducted between the 1990s and the early 2010s, with delusions again being the primary focus in the vast majority of cases. From these studies, the most consistent evidence is for right-sided frontal hypometabolism and hypoperfusion in right frontal and temporal cortices. However, whether this pattern of right-sided deficit is due to a relative increase in perfusion and/or metabolism in the left hemisphere or a reduction in the right is unclear. There is minimal evidence of perfusion change in the posterior regions or hippocampus ${ }^{24,77}$.

To our knowledge, only one SPECT study and two ${ }^{18} \mathrm{~F}$-FDG PET studies of AD psychosis have been conducted since the early $2010 \mathrm{~s}$. The two ${ }^{18} \mathrm{~F}-\mathrm{FDG}$ PET studies comprised one examination of delusions and one of hallucinations $\mathrm{s}^{88,89}$. Both studies reported right orbitofrontal hypometabolism, although this finding does not necessarily indicate that common mechanisms underlie both psychotic symptoms. As with the aforementioned atrophy study, a substantial proportion of participants in the ${ }^{18} \mathrm{~F}$-FDG PET hallucination study would be expected to have concomitant delusions, so the similar findings could reflect the presence of delusions among individuals with hallucinations.

In the newer SPECT study, regional hypoperfusion was largely confined to the right hemisphere (inferior temporal gyrus, parahippocampal cortex, posterior insula and amygdala) but was observed bilaterally in the temporal poles ${ }^{90}$. Interestingly, different subtypes of delusions were associated with different patterns of hypoperfusion. Although the sample size was small, this analysis serves to highlight the complexities around symptom classifications and underscores the need for a new framework in which to assess symptoms (see nosology section above), which could also help to explain the inconsistencies observed in previous studies.

We are only aware of two studies that used nuclear imaging to examine receptor changes associated with psychosis. One was a study of only nine patients with $\mathrm{AD}$, which found no correlation between 5-hydroxytryptamine $2 \mathrm{~A}$ receptor (5-HT2A) binding and psychosis scores on the NPI ${ }^{91}$. However, in view of the small sample size, no reliable conclusions can be drawn. The other study, which examined dopamine receptors in 23 patients with $\mathrm{AD}$, reported an increased number of striatal D2/3 receptors in individuals with psychosis ${ }^{92}$.

\section{Neurobiology}

Consistent with the evidence from neuroimaging studies, which implicated frontal dysfunction in AD psychosis, numerous post-mortem studies have found that psychotic symptoms in $\mathrm{AD}$ are associated with an increased burden of neurofibrillary tangle pathology and hyperphosphorylated $\operatorname{tau}^{24,93-96}$. Several other studies indicated that tau hyperphosphorylation was associated with psychosis in women but not in men, aligning with previous findings from cerebrospinal fluid analysis and neuroimaging as well as with clinical reports of greater psychosis risk in women than in men ${ }^{13,97,98}$. The question of whether tau pathology represents a cause of psychosis or is associated with another process remains unclear, and longitudinal analysis of tau using PET might provide a better understanding of the sequence of events. Such a study has yet to be undertaken in AD psychosis, although a study using the tau PET tracer ${ }^{11} \mathrm{C}-\mathrm{PBB} 3$ in patients with traumatic brain injury linked higher levels of binding in white matter to more severe late-onset psychosis $^{99}$. This finding, considered alongside those from post-mortem studies, would suggest that further in vivo studies of the links between tau and $\mathrm{AD}$ psychosis are warranted.

Besides tau, another key correlate of cognitive decline in $\mathrm{AD}$ is neocortical synaptic disruption, with inhibition of long-term potentiation and consequent dendritic spine loss being a candidate mechanism. Soluble amyloid- $\beta(A \beta)$ is one possible cause of synaptic disruption, with one study implicating an increased $A \beta_{42}: A \beta_{40}$ ratio in $\mathrm{AD}$ psychosis ${ }^{100}$. In addition, this study found that levels of the guanine nucleotide exchange factor kalirin, which mediates long-term potentiation, were reduced in post-mortem dorsolateral prefrontal cortex tissue from people with AD psychosis. More broadly, increased levels of synaptic proteins involved in vesicular function have been shown to confer resilience to psychosis $^{96}$. Loss of zinc transporter 3, another protein that is involved in synaptic function, is also associated with psychosis, tau pathology in the anterior cingulate, and greater cognitive impairment in $\mathrm{AD}$ and $\mathrm{DLB}^{101,102}$.

The higher prevalence of psychotic symptoms in the early stages of DLB compared with AD raises the question of whether Lewy body disease contributes to psychosis risk in AD. Although comorbid Lewy body pathology does seem to confer a risk of psychosis in $\mathrm{AD}$, it does not account for all cases. Some of the key studies outlined above controlled appropriately for $a$-synuclein pathology ${ }^{96,100}$, and psychosis in $\mathrm{AD}$ has been shown to present in the absence of any comorbid Lewy body pathology ${ }^{103}$. Comorbid vascular pathology also seems to be associated with psychotic symptoms in $\mathrm{AD}$. Two studies that examined a range of vascular pathologies, both using the NACC data set, reported that subcortical arteriosclerotic leukoencephalopathy and severe arteriosclerosis were risk factors for psychosis ${ }^{22,104}$. The association between leukoencephalopathy and psychosis in $\mathrm{AD}$ was replicated in a 12-year longitudinal study, which also implicated small vessel disease and cerebral amyloid angiopathy ${ }^{105}$. The clinical observational literature also provides a degree of support for these findings, with some ${ }^{22,106}$ but not all ${ }^{107-109}$ studies indicating that a clinical history of vascular risk factors influences the risk of psychosis.

With regard to neurotransmitter disruption, postmortem studies have shown increased D3 receptor density in the nucleus accumbens and reduced dopaminergic neurotransmission in the amygdala in individuals with $\mathrm{AD}$ psychosis - findings that have been supported by PET imaging ${ }^{92,110}$. This work has prompted efforts to optimize D2/D3 receptor occupancy for amisulpride - a D2/D3 receptor antagonist that is commonly used 
to treat psychosis - with a view to minimizing extrapyramidal side effects while optimizing the therapeutic effects ${ }^{111}$. This research could pave the way for further exploration of this drug in $\mathrm{AD}$ psychosis, as discussed below. Interestingly, computational network analysis has identified dopaminergic mechanisms that could underlie the putative beneficial effects of vitamin $\mathrm{D}$ on psychosis in $\mathrm{AD}^{112}$.

A number of other post-mortem studies (reviewed extensively elsewhere ${ }^{24}$ ) consistently implicated the disruption of serotonergic neurotransmission in psychotic symptoms in $\mathrm{AD}$ and $\mathrm{DLB}$, with findings that included reduced 5-HT levels in the ventral temporal cortex and prosubiculum and reduced neuron counts in the dorsal raphe nucleus and area CA1 of the hippocampus. This work informed the development of the highly selective 5-HT2A inverse agonist pimavanserin for the treatment of psychosis in $\mathrm{AD}^{113}$.

The cholinergic system could also represent an important treatment target for psychotic symptoms in $\mathrm{AD}$ and other dementias. Early studies implicated frontotemporal muscarinic receptor density in $\mathrm{AD}$ psychosis $^{24}$, and acetylcholinesterase activity - a marker of cholinergic innervation - has been explored more recently. Among individuals with $\mathrm{AD}$, reduced acetylcholinesterase activity was found in those with hallucinations. These findings suggest that cholinergic denervation is an additional mechanism that contributes to psychosis in $\mathrm{AD}^{114}$.

A conceptual model for psychosis in $\mathrm{AD}$, based on the mechanistic insights described above, is presented in FIG. 2.

\section{Treatment and management General non-pharmacological approaches}

Despite a robust evidence base for non-pharmacological management of BPSD, especially agitation ${ }^{115}$, scant evidence is available for psychosis-specific intervention. In general, non-pharmacological approaches are preferred as an initial approach before medication or at least concurrent with medication, provided that they are not precluded by acute safety issues ${ }^{116}$. In a broader sense, the well-known DICE method (describe, investigate, create and evaluate) demonstrates the first principles of managing behavioural changes in dementia ${ }^{117}$. The first step includes measurement-based care to describe in detail the NPS or behaviour. A medical and environmental work-up is essential to investigate the causes, so as to ensure that medical issues (for example, hearing loss, pain or infection), adverse effects of drugs (such as anticholinergics or opiates) and environmental factors (for example, noise, light or disorientation) are addressed as potential contributors to NPS. In the absence of these investigations, a diagnosis of syndromal psychosis as part of a neurodegenerative disorder cannot be made with confidence. Subsequently, a management plan is created to prevent and respond to behavioural issues. The management plan is then evaluated and modified as needed ${ }^{117,118}$. Some formal intervention approaches, such as integrated care pathways ${ }^{119}$ or the WHELD ${ }^{120}$ (Improving Wellbeing and Health for People with Dementia) programme, also incorporate the principles of medication clean-up or review, exploration of reversible causes of behavioural changes, and implementation of non-pharmacological interventions.

To our knowledge, only one published study has specifically tested non-pharmacological interventions for the treatment of psychosis in individuals with dementia $^{121}$. This study, in a Taiwanese sample of men with dementia living in veterans' homes, used a broad approach involving music therapy, orientation training, art-cognitive activities and physical activities, which was shown to reduce psychotic symptoms. In this sample, the intervention improved NPI scores of delusions, hallucinations and agitation relative to other NPS. These data support, at least in principle, the potential for non-pharmacological interventions to treat psychosis in $\mathrm{AD}$; however, additional studies are required.

In clinical practice, non-pharmacological approaches for the treatment of psychosis in $\mathrm{AD}$ are a best-practice adjunct to the use of medications when psychotic symptoms are determined to be the primary NPS ${ }^{122}$. These approaches include not confronting or arguing with a person about their delusions or attempting to 'give them reality', as such confrontation can often exacerbate a sense of fear or paranoia ${ }^{123}$. Clinically, looking at the affect behind the delusions can often be helpful. For example, if the person with dementia-related psychosis is fearful, reassuring the person that they are safe and that the caregiver is there to help them can assist in assuaging that anxiety. In addition, before prescribing medications, it is important to ascertain whether a psychotic symptom is truly distressing ${ }^{124}$. For example, some hallucinations are experienced as transient and benign (for example, seeing a small child playing) and, if no disruption to function is evident, a risk-benefit assessment might conclude that treatment is not required.

\section{Pharmacological interventions}

Antipsychotics. Historically, the mainstays for the treatment of psychosis in $\mathrm{AD}$ and related dementias have been antipsychotic medications, carried over from their use to treat psychosis and agitation in patients with other psychiatric disorders. These medications have been discussed extensively and, although modest efficacy has been established ${ }^{125}$, this efficacy comes at the cost of a substantial adverse effect burden ${ }^{4,118,126,127}$ and mortality risk ${ }^{128}$. Overall, meta-analytical evidence best supports aripiprazole and risperidone, with substantially less evidence for quetiapine and olanzapine ${ }^{125,129,130}$. Consistent with these observations, a network metaanalysis, which addressed the treatment of BPSD and not of psychosis specifically, suggested that aripiprazole was the most effective and safe atypical antipsychotic, with olanzapine providing the least benefit overall ${ }^{131}$. However, the treatment indication could also be relevant to the choice of drug. Risperidone is known to carry a risk of stroke but a post hoc analysis of clinical trial data indicated that, among patients with dementia, this risk was lower in individuals with delusions than in those with other NPS such as agitation, suggesting that a more nuanced and personalized approach could mitigate the risks associated with antipsychotic drug use ${ }^{132}$. 


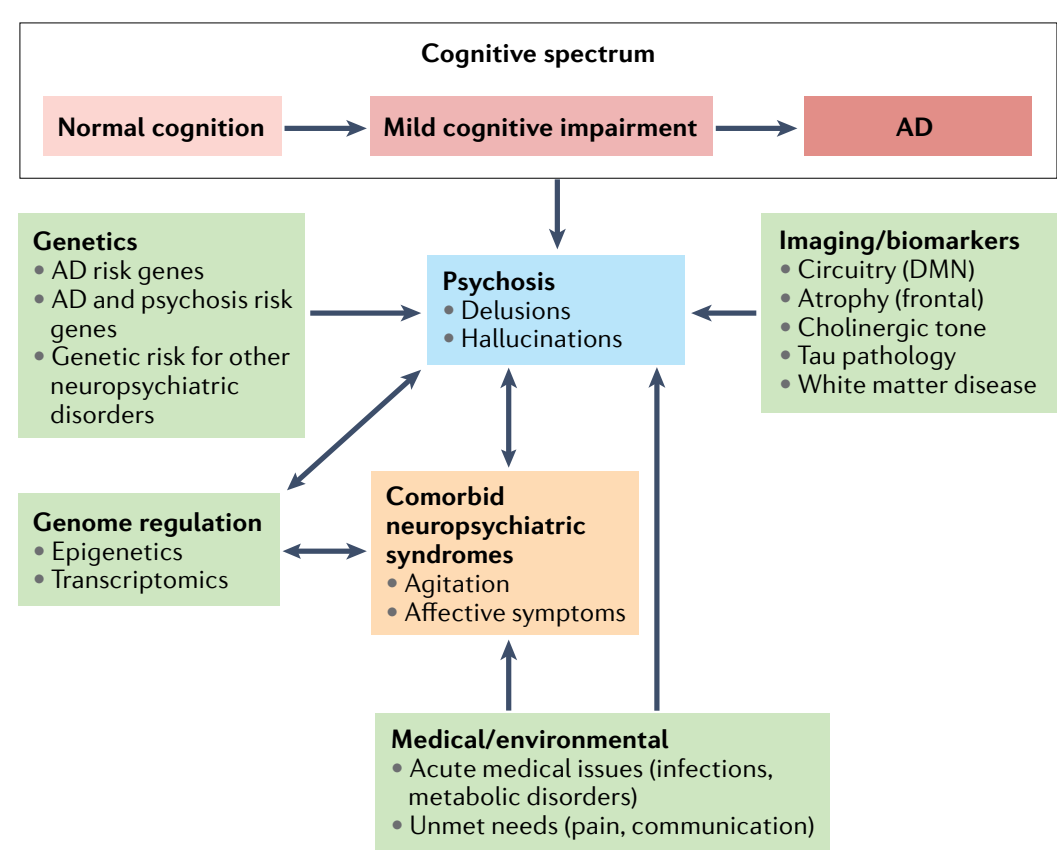

Fig. 2 | Psychosis in AD: a conceptual model. Psychosis can emerge across the cognitive continuum in older adults. Before syndromic psychosis can be diagnosed, medical and environmental issues need to be addressed, as do comorbid agitation or affective syndromes, which might be primary and require different assessment and treatment approaches. Delusions and hallucinations often coexist but can have different features and natural histories. Furthermore, persecutory and misidentification delusions probably have different aetiologies, with the latter being associated with greater hippocampal atrophy. Genetic markers include genes associated with Alzheimer disease (AD), psychosis and other neuropsychiatric disorders. Imaging and biomarker correlates include default mode network (DMN) dysfunction, cortical atrophy, cholinergic mechanisms, hyperphosphorylated tau and white matter disease.

More recent pharmacokinetic-pharmacodynamic studies have explored nuanced dosing approaches to better optimize the balance between efficacy and safety - an essential strategy in the absence of other licensed classes of drugs. These studies suggest that dose adjustments based on age and Mini-Mental State Examination scores, which are putatively required owing to the loss of bloodbrain barrier integrity ${ }^{133}$, could enable safer and more personalized use of these medications. Similarly, as described above, a series of studies has explored the approach of defining and targeting a therapeutic window of striatal D2/D3 occupancy for amisulpride in late-life psychosis. In individuals with $\mathrm{AD}$ psychosis, appropriate but higher than expected occupancy was described at very low doses, again implicating blood-brain barrier disruption ${ }^{111}$. This approach was successfully implemented in a treatment trial in people with VLOSLP ${ }^{134}$.

Other research leveraging transcriptomic data directly linked cardiovascular disease and immune system pathways to the mechanisms of action of atypical antipsychotics, suggesting that enhanced screening of cardiovascular history could inform prescribing strategies $^{135}$. Awareness of adverse drug reactions and safety issues with antipsychotics is also essential. In the USA, no medications are licensed for psychosis and agitation in dementia and, in the European Union and Canada, only risperidone is indicated. Risks include cerebrovascular accidents, extrapyramidal symptoms, falls and mortality. The risks seem to be greater with older antipsychotics than with second-generation (also known as atypical) antipsychotics ${ }^{18,128}$. Despite their relatively favourable safety profile, however, a meta-analysis found that atypical antipsychotics still carried a 3.5\% risk of mortality, representing a $54 \%$ relative increase in risk compared with the $2.3 \%$ risk for placebo ${ }^{136}$.

Novel treatments. Following FDA approval of pimavanserin for the treatment of PD psychosis ${ }^{137}$, this drug is now being explored in individuals with $\mathrm{AD}$ psychosis. Pimavanserin is a highly selective 5-HT2A inverse agonist with no dopaminergic, histaminergic or muscarinic binding ${ }^{138}$. A phase II trial, which recruited nursing home residents with $\mathrm{AD}$, demonstrated moderate efficacy in terms of a reduction in psychosis scores on the nursing home version of the NPI (Cohen's $d$ effect size 0.32 for pimavanserin over placebo at the 6 -week a priori end point $)^{113}$. A post hoc analysis in participants with more severe psychosis demonstrated a substantially larger treatment effect of drug over placebo, with an effect size of 0.73 , and a reduction in psychosis score of $\geq 30 \%$ in $88.9 \%$ of the pimavanserin-treated group compared with $43.3 \%$ of the placebo group ${ }^{139}$. A phase III relapse prevention study enroled participants with allcause dementia. Responders to pimavanserin (62\% of open-label-treated participants) were enrolled in a 6-month double-blind withdrawal phase. Relapse of psychosis was 2.8-fold lower in the drug-treated group than in the placebo group $(P=0.0023)$, and the study was halted at the pre-planned interim analysis ${ }^{140}$.

From a safety perspective, pimavanserin has no negative impact on parkinsonism, psychosis or stroke risk but is associated with modest QTc prolongation $(\sim 9 \mathrm{~ms})$ as well as confusion, oedema, falls and unstable gait. Similar to other antipsychotic drugs, pimavanserin received a black box warning for mortality risk when it was approved by the FDA for PD psychosis, with a requirement for post-marketing monitoring. No significant excess deaths were reported in the pimavanserin-treated arms (five deaths versus four in the placebo arms across the two trials $)^{113,140}$. Mortality rates are consistent with those expected for people with $\mathrm{PD}^{141}$, and the FDA has concluded that there are no new safety concerns ${ }^{142}$. However, a long-term open-label safety study in people with PD indicates that concurrent prescription with an atypical antipsychotic could have safety implications ${ }^{143}$. In 66 participants who had other antipsychotics added to their regimen, a fourfold increase in deaths and a threefold increase in serious treatment-emergent adverse events was found, suggesting that considerable caution is required if polypharmacy is being considered.

Other agents have demonstrated efficacy for psychosis in $\mathrm{AD}$ consistent with the mechanisms described above. In the CitAD study, citalopram demonstrated efficacy for reduction of the primary outcome measure of agitation, and a secondary analysis revealed a reduction in the frequency or severity of hallucinations and delusions ${ }^{144}$. However, QTc prolongation, conferred primarily by the $\mathrm{R}$-citalopram enantiomer, was also a concern with this drug, and the ongoing S-CitAD ${ }^{145}$ study is implementing 
escitalopram - the safer S-enatiomer of the racemic mix that constitutes citalopram - for the treatment of $\mathrm{AD}$ agitation, with psychosis as a secondary outcome measure. Interestingly, data published in 2020 demonstrated a reduction in cerebrospinal fluid $\mathrm{A} \beta_{42}$ levels in cognitively healthy older adults treated with escitalopram versus placebo $^{146}$, opening up potential opportunities to use this agent in dementia studies.

In the 12 -week Lit- $\mathrm{AD}^{147}$ randomized trial, low-dose lithium was assessed for efficacy in treating agitation in $\mathrm{AD}$. Although the trial was unsuccessful in meeting the primary end point of reduction in agitation and aggression scores on the NPI, the secondary outcome of improvement in clinical global impression was met. Importantly, lithium treatment was not associated with cognitive decline. Exploratory analyses determined that, compared with placebo, lithium was associated with a significant reduction in delusions $(P=0.04$, Cohen's $d 0.76$ ) but not in hallucinations ( $P=0.56$, Cohen's $d-0.21$ ). Despite limitations in sample size and study power, the separation of delusions and hallucinations, especially with respect to the substantially different effect sizes, is of interest. These findings suggest that hallucinations and delusions have different substrates and might need to be considered as separate entities. No consensus has yet been reached on how lithium works to achieve neuroprotection and clinical effects. However, glycogen synthase kinase $3 \mathrm{~B}$ (modulating apoptosis and neuroplasticity), neurotrophic factors (for example, brain-derived neurotrophic factor), neurotransmitter modulation (glutamate, dopamine, GABA, acetylcholine and glycine), and antioxidant effects have all been implicated (reviewed elsewhere ${ }^{148}$ ). Some of these mechanisms, especially the neurotransmitter effects, are consistent with what is known about psychosis in $\mathrm{AD}$. In view of the robust findings to date, particularly with regard to delusions, lithium warrants further investigation in a trial with $\mathrm{AD}$ psychotic symptoms as a primary outcome measure. Such a study could also be hypothesis generating for the further exploration of novel mechanisms as treatment targets.

Cholinesterase inhibitors are well established for the treatment of cognitive symptoms in $\mathrm{AD}$, but the therapeutic role - if any - of these medications in people with $\mathrm{AD}$ psychosis is unclear and no randomized controlled trials have been conducted. However, in an analysis from the Swedish Dementia Registry, use of cholinesterase inhibitors was associated with a reduced risk of antipsychotic initiation in the AD subgroup ${ }^{149}$. Although this observation does not confirm a treatment effect, these data are consistent with the cholinergic mechanisms that have been described in $\mathrm{AD}$ psychosis and possibly suggest an alternative to burdensome psychiatric medications in the management of NPS. Again, prospective trials are required.

Researchers are beginning to leverage the association between vitamin D deficiency and neurodegenerative disease to explore a possible role for vitamin $\mathrm{D}$ deficiency in AD psychosis. Among individuals with $\mathrm{MCI}$ or $\mathrm{AD}$, vitamin $\mathrm{D}$ use was found to be more frequent in those without psychosis and was also associated with delayed time to psychosis ${ }^{150}$. As a safe and low-cost intervention, vitamin D merits further exploration in this population.

\section{Mild behavioural impairment}

Since the time of Alois Alzheimer and publication of the case of August D., NPS have been known to emerge in advance of $\mathrm{AD}^{151}$; however, owing to the prevailing cognocentric paradigm of $\mathrm{AD}$, this notion has been underappreciated. Nevertheless, growing epidemiological evidence is leading to an increased appreciation that all-cause dementia, including $\mathrm{AD}$, can have a behavioural prodrome ${ }^{152}$. Emerging evidence also indicates that $\mathrm{AD}$ neurodegenerative pathology can result in NPS in advance of cognitive decline ${ }^{153-156}$. One study identified delusions at baseline as a predictor of amyloid positivity in a pooled diagnostic sample, and new-onset delusions and hallucinations were associated with amyloid burden in people with $\mathrm{MCI}^{157}$. Similarly, a study in a small but epidemiologically representative Finnish cohort aged $\geq 60$ years found a cross-sectional association between first-episode psychosis and cortical atrophy ${ }^{158}$. These data further support a biological link between AD proteinopathies and NPS, highlighting the need to revisit nosological constructs around late-life psychiatric symptomatology.

To attempt to reconcile the literature on the association between late-onset NPS and dementia and to standardize the approach to nomenclature and neuroscience, ISTAART have developed research diagnostic criteria for mild behavioural impairment $(\mathrm{MBI})^{159}$. MBI is a validated syndrome characterized by late onset of persistent NPS, representing a change from longstanding patterns of behaviour, as an at-risk state for incident cognitive decline and dementia. The key to dementia prognostication lies in the stipulation that MBI symptoms present de novo in later life as emergent symptoms carry a greater risk of cognitive decline and show a different trajectory compared with psychiatric disorders that recur in later life ${ }^{160}$. ISTAART have also developed a rating scale for MBI case ascertainment, known as the MBI checklist (MBI-C) $)^{161}$, which was designed to capture emergent and persistent symptoms in functionally independent community-dwelling populations. The MBI-C has been validated in community and clinical settings and across preclinical and prodromal disease populations ${ }^{162-167}$.

The behavioural and personality changes that characterize MBI can emerge at any point along the pre-dementia cognitive spectrum from normal cognition through subjective cognitive decline (SCD) to MCI. Psychotic symptoms represent one of five MBI domains. Epidemiologically, MBI is frequent, with prevalence estimates varying depending on the nature of the population and the case ascertainment approach (TABLE 2). The MBI-C has generated the most conservative prevalence estimates, ranging from $6-11 \%$ in individuals with normal cognition or SCD ${ }^{162,164,166,168}$ to $14-26 \%$ in individuals with $\mathrm{MCI}^{164,165}$. Consistent with the previous literature ${ }^{169}$, cases are over-represented in clinical versus community samples. Longitudinal cohorts have demonstrated associations between $\mathrm{MBI}$ and cognitive decline, incident MCI, and incident dementia ${ }^{160,162,167,170,171}$ and MBI has also been linked to known dementia markers, 
Table 2 | Frequency of psychosis across the cognitive spectrum

\begin{tabular}{|c|c|c|}
\hline Cognitive status & Phenomenology & Prevalence \\
\hline $\begin{array}{l}\text { Late-onset psychosis assessed } \\
\text { in the mild behavioural } \\
\text { impairment framework in } \\
\text { people with normal cognition }\end{array}$ & $\begin{array}{l}\text { Only one study conducted; suspicious } \\
\text { thoughts were the prevailing symptom; } \\
\text { other symptoms were present but at very } \\
\text { low frequencies }\end{array}$ & $\begin{array}{l}5 \% \text { if rated by proxy, } 2.5 \% \text { if self-rated } \\
\text { (suspicious thoughts } \sim 2-4 \% \text {, visual } \\
\text { hallucinations, auditory hallucinations, } \\
\text { grandiose delusions and persecutory } \\
\text { delusions } 0.1-0.8 \% \text { ) }\end{array}$ \\
\hline Mild cognitive impairment & $\begin{array}{l}\text { Persecutory delusions and visual } \\
\text { hallucinations are the most common } \\
\text { symptoms }\end{array}$ & $\begin{array}{l}\text { Delusions } 3.1-10.5 \% \text {, hallucinations } \\
1.3-2.6 \%\end{array}$ \\
\hline Dementia & $\begin{array}{l}\text { Persecutory delusions of theft, spousal } \\
\text { infidelity. and personal harm and } \\
\text { misidentification; first-rank symptoms } \\
\text { (for example, thought insertion, withdrawal } \\
\text { and broadcasting) are very rare; } \\
\text { hallucinations are predominantly visual } \\
\text { and involve people, animals or objects }\end{array}$ & $\begin{array}{l}41 \% \text { ( } 23 \% \text { delusions only, } 5 \% \\
\text { hallucinations only, } 13 \% \text { both) }\end{array}$ \\
\hline
\end{tabular}

including $A \beta^{172,173}$, tau ${ }^{174,175}$, white matter atrophy ${ }^{176}$, grey matter atrophy ${ }^{177}$, plasma neurofilament light ${ }^{178}$ and $\mathrm{AD}$ risk loci ${ }^{179,180}$.

Data on MBI psychosis are still emerging. Using the MBI-C, the prevalence of MBI psychosis in a large community sample was calculated at $3-6 \%{ }^{163}$, whereas in a cognitive neurology clinic sample the prevalence was $5.4 \%$ in people with SCD and $17.2 \%$ in people with $\mathrm{MCI}^{164}$. Longitudinal data for psychosis are scant at this stage. In one study of 234 individuals with $\mathrm{MCI}^{43}$, psychotic symptoms (measured with the NPI-Q - a brief informant-reported form of the NPI) were present in 3\% of the cohort and the prevalence was disproportionately high in those who progressed to dementia within 3 years (6.3\% versus $0.8 \%$ for non-progressors, $P=0.045$ ). Psychosis was the only MBI domain that showed a significant association with dementia, with a hazard ratio of 2.9. In a subsequent longitudinal analysis of 8,181 otherwise cognitively healthy participants in the PROTECT study (currently in preprint ${ }^{181}$ ), the prevalence of MBI-C-measured psychosis was comparable between men and women $(5.7 \%$ and $6.8 \%$, respectively, $P=0.09$ ). Over a median follow-up period of 3.07 years, global MBI burden and all MBI domains were associated with cognitive decline. Of all the domains, the association with psychosis had the largest effect size for cognitive decline but only in men. This intriguing finding warrants further exploration, not only for emergent psychosis in older adults but also for sex differences in risk, which might further inform research on sex-specific interventions.

The emerging data indicate that individuals with MBI represent a group at considerable risk of cognitive decline and dementia and that, of the MBI domains, psychosis seems to be one of the least frequent but carries the highest risk of cognitive decline. More research with larger samples to ensure adequate power is required to link AD biomarkers to psychosis. Such studies, harmonized with the new ISTAART psychosis criteria, could also offer an approach to precision medicine. With earlier identification of older adults at risk, in advance of overt symptoms or risks to personal safety, this approach could provide an opportunity to explore current and novel medications for the management of late-life emergent psychiatric symptoms and potentially delay or prevent incident dementia ${ }^{182}$.

\section{Conclusions and future challenges}

Over the past decade, several important milestones have been reached in the clinical assessment and management of psychosis and other NPS in dementia. These milestones include recognition of the substantial risk associated with antipsychotic drugs, with a resultant reduction in prescriptions ${ }^{183}$; a greater focus on non-pharmacological interventions (for example, DICE or WHELD); exploration of strategies to optimize existing drugs, such as aripiprazole, risperidone, amisulpride and escitalopram, for safer precision-based treatment; development of a clinical trial programme for pimavanserin; and publication of new consensus definitions and criteria that better reflect the emergence of psychosis in advance of dementia at preclinical and prodromal stages (for example, IPA Psychosis, ISTAART-AA Psychosis, and ISTAART-AA MBI criteria). However, the impact of these milestones is limited by the lack of a licensed pharmacological therapy, the relatively sparse drug development pipeline, the continued use of antipsychotics despite the risks, and the dearth of any specific non-pharmacological intervention for psychosis.

In the absence of approved medications, the development of precise risk and implementation profiles for existing antipsychotics is an essential and immediate goal. Clinical studies, along with statistical and computational modelling, have identified aripiprazole as the antipsychotic medication that best balances efficacy and safety. This work has also explored precision approaches to implement risperidone and amisulpride for psychosis in $\mathrm{AD}$, which could improve the safety profile of these agents. These approaches can be extended to different settings to assist in the development of evidence-based algorithms, involving both pharmacological and non-pharmacological interventions, that are tailored to specific patient profiles. Non-pharmacological modalities need to be developed and assessed specifically for psychosis and, as highlighted by the COVID-19 pandemic, they should ideally be suitable for both in-person and virtual use. 
Concurrent exploration of the neurobiology of psychosis could inform the development of novel agents or repurposing of existing agents. Research into the underlying neurobiology has identified the first genome-wide significant loci and the first evidence of DNA methylation changes in $\mathrm{AD}$ psychosis, although further replication and functional validation are required. Meanwhile, the evidence from neuropathology and imaging studies has cemented frontal dysfunction and tau pathology as key neurobiological correlates of psychosis. Synaptic dysfunction and the protein kalirin are potential novel treatment targets that are worthy of further characterization. Of note, most agents in development for AD psychosis have mechanisms of action that are related to older antipsychotics first developed for schizophrenia, possibly indicating common underlying disease mechanisms. Evidence implicating the DMN, genomic and epigenomic risk factors for schizophrenia and depression, and kalirin and vitamin $\mathrm{D}$ in $\mathrm{AD}$ psychosis suggest some mechanistic overlap with schizophrenia. One option for future studies would be the more systematic examination of the symptoms of psychosis in $\mathrm{AD}$ and their mechanistic overlap with those of schizophrenia. This approach could facilitate the more targeted use of antipsychotics, as one might hypothesize that the existence of a schizophrenia-like phenotype would confer a better response to antipsychotics, and alternative treatment approaches could be considered for symptom profiles less consistent with schizophrenia.
To facilitate symptom profile-based approaches, better phenomenology and nosology need to be implemented during case ascertainment of $\mathrm{AD}$ psychosis. The evolution of the IPA psychosis criteria and the development of the ISTAART psychosis and MBI criteria should aid this effort. In fact, the ISTAART psychosis criteria were developed explicitly with these goals in mind. These criteria place late-life emergent psychosis on a spectrum, incorporating the natural history of symptoms, symptom modality, cognitive stages and AD biomarkers in order to frame psychotic symptoms. The ISTAART criteria were also intended to foster genetics research, offering classification approaches that can be tailored to the analyses implemented (for example, splitting delusions and hallucinations and ensuring more non-psychosis 'controls' by incorporating past history). Cross-sectional and longitudinal studies could incorporate this framework to help us better understand the clinical, cognitive and neurobiological correlates of $\mathrm{AD}$ psychosis and to aid drug development and repurposing as well as the development and implementation of non-pharmacological interventions.

$\mathrm{AD}$ psychosis remains an important public health issue, affecting individuals with dementia and their caregivers at a personal level. However, as this Review highlights, progress is being made and the next waves of research should provide more refined and targeted approaches to managing this syndrome.

Published online 4 January 2022
1. Steinberg, M. et al. Point and 5-year period prevalence of neuropsychiatric symptoms in dementia: the Cache County Study. Int. J. Geriatr. Psychiatry 23, 170-177 (2008).

2. Lanctôt, K. L. et al. Neuropsychiatric signs and symptoms of Alzheimer's disease: new treatment paradigms. Alzheimers Dement. 3, 440-449 (2017).

3. Lyketsos, C. G. et al. Neuropsychiatric symptoms in Alzheimer's disease. Alzheimers Dement. 7, 532-539 (2011)

4. Creese, B., Da Silva, M. V., Johar, I. \& Ballard, C The modern role of antipsychotics for the treatment of agitation and psychosis in Alzheimer's disease. Expert Rev. Neurother. 18, 461-467 (2018).

5. Ballard, C. G. et al. Management of agitation and aggression associated with Alzheimer disease. Nat. Rev. Neurol. 5, 245-255 (2009).

6. Watt, J. A. et al. Safety of pharmacologic interventions for neuropsychiatric symptoms in dementia: a systematic review and network meta-analysis. BMC Geriatr. 20, 212 (2020).

7. Kaufer, D. I. et al. Assessing the impact of neuropsychiatric symptoms in Alzheimer's disease: the Neuropsychiatric Inventory Caregiver Distress Scale. J. Am. Geriatr. Soc. 46, 210-215 (1998)

8. Wiener, P. K., Kiosses, D. N., Klimstra, S., Murphy, C. \& Alexopoulos, G. S. A short-term inpatient program for agitated demented nursing home residents. Int. J. Geriatr. Psychiatry 16, 866-872 (2001)

9. Fischer, C. E., Ismail, Z. \& Schweizer, T. A. Delusions increase functional impairment in Alzheimer's disease. Dement. Geriatr. Cogn. Disord. 33, 393-399 (2012).

10. Wilson, R. et al. Hallucinations, cognitive decline, and death in Alzheimer's disease. Neuroepidemiology 26, 68-75 (2006)

11. Scarmeas, N. et al. Delusions and hallucinations are associated with worse outcome in Alzheimer disease. Arch. Neurol. 62, 1601-1608 (2005)

12. Zahodne, L. B., Ornstein, K., Cosentino, S. Devanand, D. P. \& Stern, Y. Longitudinal relationships between Alzheimer disease progression and psychosis, depressed mood, and agitation/aggression. Am. J. Geriatr. Psychiatry 23, 130-140 (2015).

13. Ropacki, S. A. \& Jeste, D. V. Epidemiology of and risk factors for psychosis of Alzheimer's disease: a review of 55 studies published from 1990 to 2003. Am. J. Psychiatry 162, 2022-2030 (2005).
14. Bassiony, M. M. et al. Delusions and hallucinations in Alzheimer's disease: prevalence and clinical correlates. Int. J. Geriatr. Psychiatry 15, 99-107 (2000).

15. Qian, W., Fischer, C. E., Schweizer, T. A. \& Munoz, D. G Association between psychosis phenotype and APOE genotype on the clinical profiles of Alzheimer's disease Curr. Alzheimer Res. 15, 187-194 (2018).

16. Holt, A. E. \& Albert, M. L. Cognitive neuroscience of delusions in aging. Neuropsychiatr. Dis. Treat. 2 , 181 (2006).

17. Ismail, Z. et al. Neurobiology of delusions in Alzheimer's disease. Curr. Psychiatry Rep. 13, 211-218 (2011).

18. Cummings, J. et al. Pimavanserin: potential treatment for dementia-related psychosis. J. Prev. Alzheimers Dis. 5, 253-258 (2018)

19. Vik-Mo, A. O., Giil, L. M., Ballard, C. \& Aarsland, D. Course of neuropsychiatric symptoms in dementia: 5-year longitudinal study. Int. J. Geriatr. Psychiatry 33, 1361-1369 (2018)

20. Vik-Mo, A. O., Giil, L. M., Borda, M. G., Ballard, C. $\&$ Aarsland, D. The individual course of neuropsychiatric symptoms in people with Alzheimer's and Lewy body dementia: 12-year longitudinal cohort study. Br. J. Psychiatry 216, 43-48 (2020).

21. Fischer, C. E. et al. Determining the impact of psychosis on rates of false-positive and false-negative diagnosis in Alzheimer's disease. Alzheimers Dement. 3, 385-392 (2017).

22. Fischer, C. E. et al. Lewy bodies, vascular risk factors, and subcortical arteriosclerotic leukoencephalopathy, but not Alzheimer pathology, are associated with development of psychosis in Alzheimer's disease. J. Alzheimers Dis. 50, 283-295 (2016).

23. Rubin, E. H., Drevets, W. C. \& Burke, W. J. The nature of psychotic symptoms in senile dementia of the Alzheimer type. J. Geriatr. Psychiatry Neurol. 1, 16-20 (1988).

24. Murray, P. S. Kumar, S., DeMichele-Sweet, M. A. A $\&$ Sweet, R. A. Psychosis in Alzheimer's disease. Biol. Psychiatry 75, 542-552 (2014).

25. Nagahama, Y. et al. Prevalence and clinical implications of the mirror and TV signs in advanced Alzheimer's disease and dementia with Lewy bodies. Dement. Geriatr. Cogn. Dis. Extra 10, 56-62 (2020).

26. Roane, D. M., Feinberg, T. E. \& Liberta, T. A. Delusional misidentification of the mirror image. Curr. Neurol. Neurosci. Rep. 19, 55 (2019).
27. Feinberg, T. E. \& Roane, D. Self-representation in delusional misidentification and confabulated "others". Cortex 87, 118-128 (2017).

28. El Haj, M. et al. Clinical and neurocognitive aspects of hallucinations in Alzheimer's disease. Neurosci. Biobehav. Rev. 83, 713-720 (2017).

29. Ballard, C. \& Oyebode, F. Psychotic symptoms in patients with dementia. Int. J. Geriatr. Psychiatry 10, 743-752 (1995).

30. Jeste, D. V. \& Finkel, S. I. Psychosis of Alzheimer's disease and related dementias: diagnostic criteria for a distinct syndrome. Am. J. Geriatr. Psychiatry $\mathbf{8}$, 29-34 (2000)

31. Cohen-Mansfield, J. \& Golander, H. The measurement of psychosis in dementia: a comparison of assessment tools. Alzheimer Dis. Assoc. Disord. 25, 101-108 (2011).

32. Ismail, Z. et al. A comparison of the E-BEHAVE-AD, NBRS, and NPI in quantifying clinical improvement in treatment of agitation and psychosis associated with dementia. Am. J. Geriatr. Psychiatry 21, 78-87 (2013).

33. Monastero, R., Mangialasche, F., Camarda, C. Ercolani, S. \& Camarda, R. A systematic review of neuropsychiatric symptoms in mild cognitive impairment. J. Alzheimers Dis. 18, 11-30 (2009).

34. Cummings, J. et al. Criteria for psychosis in major and mild neurocognitive disorders: International Psychogeriatric Association (IPA) consensus clinical and research definition. Am. J. Geriatr. Psychiatry 28 1256-1269 (2020).

35. Schneider, L. S. et al. Psychosis of Alzheimer disease: validity of the construct and response to risperidone. Am. J. Geriatr. psychiatry 11, 414-425 (2003).

36. Cummings, J. et al. Agitation in cognitive disorders: International Psychogeriatric Association provisional consensus clinical and research definition. Int. Psychogeriatr. 27, 7-17 (2015).

37. De Mauleon, A. et al. Agitation in Alzheimer's disease novel outcome measures reflecting the International Psychogeriatric Association (IPA) agitation criteria. Alzheimers Dement. 17, 1687-1697 (2021).

38. Aalten, P et al. Neuropsychiatric syndromes in dementia. Dement. Geriatr. Cogn. Disord. 24, 457-463 (2007)

39. Siafarikas, N. et al. Frequency and subgroups of neuropsychiatric symptoms in mild cognitive 
impairment and different stages of dementia in Alzheimer's disease. Int. Psychogeriatr. 30, 103-113 (2018)

40. Liew, T. M. Symptom clusters of neuropsychiatric symptoms in mild cognitive impairment and their comparative risks of dementia: a cohort study of 8530 older persons. J. Am. Med. Dir. Assoc. 20, 1054.e1-1054.e9 (2019).

41. Martin, E. \& Velayudhan, L. Neuropsychiatric symptoms in mild cognitive impairment: a literature review. Dement. Geriatr. Cogn. Disord. 49, 146-155 (2020).

42. Peters, M. E. et al. Neuropsychiatric symptoms as risk factors for progression from CIND to dementia: the Cache County Study. Am. J. Geriatr. Psychiatry 21, 1116-1124 (2013)

43. Yokoi, Y. et al. Discrete effect of each mild behavioural impairment category on dementia conversion or cognitive decline in patients with mild cognitive impairment. Psychogeriatrics 19, 591-600 (2019).

44. Dietlin, S. et al. Neuropsychiatric symptoms and risk of progression to Alzheimer's disease among mild cognitive impairment subjects. J. Alzheimers Dis. $\mathbf{7 0}$ 25-34 (2019)

45. Rosenberg, P. B. et al. The association of neuropsychiatric symptoms in $\mathrm{MCl}$ with incident dementia and Alzheimer disease. Am. J. Geriatr. Psychiatry 21, 685-695 (2013).

46. Pink, A. et al. Neuropsychiatric symptoms, APOE epsilon 4, and the risk of incident dementia: a population-based study. Neurology 84, 935-943 (2015)

47. Fischer, C. E. \& Agüera-Ortiz, L. Psychosis and dementia: risk factor, prodrome, or cause? Int. Psychogeriatr. 30, 209-219 (2018).

48. Lapid, M. I. \& Ho, J. B. Challenging our beliefs about delusional disorder in late life. Int. Psychogeriatr. 32 423-425 (2020)

49. Nagendra, J. \& Snowdon, J. An Australian study of delusional disorder in late life. Int. Psychogeriatr. 32, 453-462 (2020)

50. Brodaty, H., Sachdev, P., Koschera, A., Monk, D. $\&$ Cullen, B. Long-term outcome of late-onset schizophrenia: 5-year follow-up study. Br. J. Psychiatry 183, 213-219 (2003).

51. Kohler, S. et al. Cognitive decline and dementia risk in older adults with psychotic symptoms: a prospective cohort study. Am. J. Geriatr. Psychiatry 21, 119-128 (2013)

52. Soares, W. B., dos Santos, E. B., Bottino, C. M. D. C. $\&$ Elkis, H. Psychotic symptoms in older people without dementia from a Brazilian community-based sample: a seven years' follow-up. PLoS One 12 , e0178471 (2017).

53. Liew, T. M. Neuropsychiatric symptoms in cognitively normal older persons, and the association with Alzheimer's and non-Alzheimer's dementia. Alzheimers Res. Ther. 12, 35 (2020).

54. Korner, A., Lopez, A. G., Lauritzen, L., Andersen, P. K. $\S$ Kessing, L. V. Late and very-late first-contact schizophrenia and the risk of dementia - a nationwide register based study. Int. J. Geriatr. Psychiatry $\mathbf{2 4}$ 61-67 (2009).

55. Kørner, A., Lopez, A. G., Lauritzen, L., Andersen, P. K. $\&$ Kessing, L. V. Acute and transient psychosis in old age and the subsequent risk of dementia: a nationwide register-based study. Geriatr. Gerontol. Int. 9, 62-68 (2009).

56. Almeida, O. P. et al. Risk of dementia associated with psychotic disorders in later life: the health in men study (HIMS). Psychol. Med. 49, 232-242 (2019)

57. Stafford, J. et al. Association between risk of dementia and very late-onset schizophrenia-like psychosis a Swedish population-based cohort study. Psychol. Med. https://doi.org/10.1017/S0033291721002099 (2021).

58. Nagao, S. et al. Argyrophilic grain disease as a neurodegenerative substrate in late-onset schizophrenia and delusional disorders. Eur. Arch. Psychiatry Clin. Neurosci. 264, 317-331 (2014).

59. Fischer, C. E. et al. Revisiting criteria for psychosis in Alzheimer's disease and related dementias: toward better phenotypic classification and biomarker research. J. Alzheimers Dis. 73, 1143-1156 (2020).

60. Bacanu, S.-A. et al. Heritability of psychosis in Alzheimer disease. Am. J. Geriatr. Psychiatry 13, 624-627 (2005)

61. DeMichele-Sweet, M. A. A. et al. Genome-wide association identifies the first risk loci for psychosis in Alzheimer disease. Mol. Psychiatry https://doi.org/ 10.1038/s41380-021-01152-8 (2021).
62. Shah, C., DeMichele-Sweet, M. A. A. \& Sweet, R. A Genetics of psychosis of Alzheimer disease. Am. J. Med. Genet. B Neuropsychiatr. Genet. 174, 27-35 (2017).

63. Kim, J., Fischer, C. E., Schweizer, T. A. \& Munoz, D. G. Gender and pathology-specific effect of Apolipoprotein E genotype on psychosis in Alzheimer's disease. Curr. Alzheimer Res. 14, 834-840 (2017).

64. DeMichele-Sweet, M. A. A. et al. Genetic risk for schizophrenia and psychosis in Alzheimer disease. Mol. Psychiatry 23, 963-972 (2018).

65. Creese, B. et al. Examining the association between genetic liability for schizophrenia and psychotic symptoms in Alzheimer's disease. Transl. Psychiatry 9 273 (2019).

66. Legge, S. E et al. Association of genetic liability to psychotic experiences with neuropsychotic disorder and traits. JAMA Psychiatry 76, 1256-1265 (2019).

67. Ellis, N. et al. Genetic risk underlying psychiatric and cognitive symptoms in Huntington's Disease. Biol. Psychiatry 87, 857-865 (2020)

68. Pain, O. et al. Genome-wide analysis of adolescent psychotic-like experiences shows genetic overlap with psychiatric disorders. Am. J. Med. Genet. B Neuropsychiatr. Genet. 177, 416-425 (2018).

69. Bebbington, P. \& Freeman, D. Transdiagnostic extension of delusions: schizophrenia and beyond. Schizophr. Bull. 43, 273-282 (2017).

70. Golan, D., Lander, E. S. \& Rosset, S. Measuring missing heritability: inferring the contribution of common variants. Proc. Natl Acad. Sci. USA 111 E5272-E5281 (2014).

71. Lunnon, K. et al. Methylomic profiling implicates cortical deregulation of ANK1 in Alzheimer's disease. Nat. Neurosci. 17, 1164-1170 (2014).

72. Smith, R. G. et al. Elevated DNA methylation across a 48-kb region spanning the HOXA gene cluster is associated with Alzheimer's disease neuropathology. Alzheimers Dement. 14, 1580-1588 (2018)

73. Viana, J. et al. Schizophrenia-associated methylomic variation: molecular signatures of disease and polygenic risk burden across multiple brain regions. Hum. Mol. Genet. 26, 210-225 (2017).

74. Pishva, E. et al. Psychosis-associated DNA methylomi variation in Alzheimer's disease cortex. Neurobiol. Aging 89, 83-88 (2020).

75. Creese, B. et al. Glucocerebrosidase mutations and neuropsychiatric phenotypes in Parkinson's disease and Lewy body dementias: review and meta-analyses. Am. J. Med. Genet. B Neuropsychiatr. Genet. 177, 232-241 (2018)

76. Shiner, T. et al. High frequency of GBA gene mutations in dementia with Lewy bodies among Ashkenazi Jews. JAMA Neurol. 73, 1448-1453 (2016).

77. Ismail, Z., Nguyen, M. Q., Fischer, C. E., Schweizer, T. A \& Mulsant, B. H. Neuroimaging of delusions in Alzheimer's disease. Psychiatry Res. 202, 89-95 (2012).

78. Qian, W. et al. Gray matter changes associated with the development of delusions in Alzheimer disease. Am. J. Geriatr. Psychiatry 27, 490-498 (2019).

79. Lee, K. et al. Right hippocampus atrophy is independently associated with Alzheimer's disease with psychosis. Psychogeriatrics 19, 105-110 (2019).

80. McLachlan, E., Bousfield, J., Howard, R. \& Reeves, S. Reduced parahippocampal volume and psychosis symptoms in Alzheimer's disease. Int. J. Geriatr. Psychiatry 33, 389-395 (2018).

81. Förstl, H., Burns, A., Levy, R. \& Cairns, N Neuropathological correlates of psychotic phenomena in confirmed Alzheimer's disease. Br. J. Psychiatry 165, 53-59 (1994).

82. Donovan, N. J. et al. Regional cortical thinning predicts worsening apathy and hallucinations across the Alzheimer disease spectrum. Am. J. Geriatr. Psychiatry 22, 1168-1179 (2014)

83. Fischer, C. E. et al. Gray matter atrophy in patients with mild cognitive impairment/Alzheimer's disease over the course of developing delusions. Int. J. Geriatr Psychiatry 31, 76-82 (2016).

84. Balthazar, M. L. et al. Neuropsychiatric symptoms in Alzheimer's disease are related to functional connectivity alterations in the salience network. Hum. Brain Mapp. 35, 1237-1246 (2014).

85. Lee, J. S., Kim, J. H. \& Lee, S.-K. The relationship between neuropsychiatric symptoms and default-mode network connectivity in Alzheimer's disease. Psychiatry Investig. 17, 662 (2020).

86. Qian, W. et al. Delusions in Alzheimer disease are associated with decreased default mode network functional connectivity. Am. J. Geriatr. Psychiatry 27, 1060-1068 (2019).
87. Tu, M.-C et al. Comparison of neuropsychiatric symptoms and diffusion tensor imaging correlates among patients with subcortical ischemic vascular disease and Alzheimer's disease. BMC Neurol. 17, 144 (2017).

88. Blanc, F. et al. Right anterior insula: core region of hallucinations in cognitive neurodegenerative diseases. PLoS One 9, e114774 (2014).

89. Sultzer, D. L. et al. Neurobiology of delusions, memory, and insight in Alzheimer disease. Am. J. Geriatr. Psychiatry 22, 1346-1355 (2014)

90. Nakatsuka, M. et al. Content of delusional thoughts in Alzheimer's disease and assessment of contentspecific brain dysfunctions with BEHAVE-AD-FW and SPECT. Int. Psychogeriatr. 25, 939-948 (2013).

91. Santhosh, L. et al. Regional distribution and behaviora correlates of 5-HT2A receptors in Alzheimer's disease with [18F] deuteroaltanserin and PET. Psychiatry Res. 173, 212-217 (2009)

92. Reeves, S., Brown, R., Howard, R. \& Grasby, P. Increased striatal dopamine (D2/D3) receptor availability and delusions in Alzheimer disease. Neurology 72, 528-534 (2009).

93. Koppel, J. et al. Psychotic Alzheimer's disease is associated with gender-specific tau phosphorylation abnormalities. Neurobiol. Aging 35, 2021-2028 (2014).

94. Murray, P. S. et al. Hyperphosphorylated tau is elevated in Alzheimer's disease with psychosis. J. Alzheimers Dis. 39, 759-773 (2014).

95. Ehrenberg, A. J. et al. Neuropathologic correlates of psychiatric symptoms in Alzheimer's disease. J. Alzheimers Dis. 66, 115-126 (2018)

96. Krivinko, J. M. et al. Synaptic proteome compensation and resilience to psychosis in Alzheimer's disease. Am. J. Psychiatry 175, 999-1009 (2018).

97. Whitehead, D. et al. Frontotemporal atrophy associated with paranoid delusions in women with Alzheimer's disease. Int. Psychogeriatr. 24, 99-107 (2012).

98. Koppel, J. et al. Elevated CSF Tau is associated with psychosis in Alzheimer's disease. Am. J. Psychiatry 170, 1212-1213 (2013)

99. Takahata, K. et al. PET-detectable tau pathology correlates with long-term neuropsychiatric outcomes in patients with traumatic brain injury. Brain 142 3265-3279 (2019).

100. Murray, P. S. et al. $\beta$-Amyloid $42 / 40$ ratio and kalirin expression in Alzheimer disease with psychosis Neurobiol. Aging 33, 2807-2816 (2012).

101. Whitfield, D. R., Francis, P. T., Ballard, C. \& Williams, G. Associations between ZnT3, tau pathology, agitation, and delusions in dementia. Int. J. Geriatr. Psychiatry 33, 1146-1152 (2018)

102. Whitfield, D. R. et al. Assessment of ZnT3 and PSD95 protein levels in Lewy body dementias and Alzheimer's disease: association with cognitive impairment. Neurobiol. Aging 35, 2836-2844 (2014).

103. Tsuang, D. et al. Predicting lewy body pathology in a community-based sample with clinical diagnosis of Alzheimer's disease. J. Geriatr. Psychiatry Neurol. 19, 195-201 (2006)

104. Ting, S. K. S., Hao, Y., Chia, P. S., Tan, E.-K. $\&$ Hameed, S. Clinicopathological correlation of psychosis and brain vascular changes in Alzheimer's disease. Sci. Rep. 6, 20858 (2016)

105. Vik-Mo, A. O., Bencze, J., Ballard, C., Hortobágyi, T. \& Aarsland, D. Advanced cerebral amyloid angiopathy and small vessel disease are associated with psychosis in Alzheimer's disease. J. Neurol. Neurosurg. Psychiatry 90, 728-730 (2019)

106. Kim, J., Schweizer T. A., Fischer C. E. \& Munoz, D. G. The role of cerebrovascular disease on cognitive and functional status and psychosis in severe Alzheimer's disease. J. Alzheimers Dis. 55, 381-389 (2017)

107. Steinberg, M. et al. Vascular risk factors and neuropsychiatric symptoms in Alzheimer's disease: the Cache County Study. Int. J. Geriatr. Psychiatry 29 , 153-159 (2014)

108. Bidzan, M., Bidzan, L. \& Pachalska, M. Neuropsychiatric symptoms in patients with Alzheimer's disease with a vascular component. Ann. Agric. Environ. Med. 21 412-415 (2014)

109. Hwang, I., Baek, K.-H., Han, J. H., Ha, S.-W. \& Yang, Y. Hypertension and neuropsychiatric symptoms in patients with drug-naïve Alzheimer's disease. Dement. Neurocogn. Disord. 16, 78-82 (2017).

110. Sweet, R. A. et al. Alterations of striatal dopamine receptor binding in Alzheimer disease are associated with Lewy body pathology and antemortem psychosis Arch. Neurol. 58, 466-472 (2001). 
111. Reeves, S. et al. Therapeutic window of dopamine D2/3 receptor occupancy to treat psychosis in Alzheimer's disease. Brain 140, 1117-1127 (2017).

112. Fan, P., Qi, X., Sweet, R. A. \& Wang, L. Network systems pharmacology-based mechanism study on the beneficial effects of vitamin $D$ against psychosis in Alzheimer's disease. Sci. Rep. 10, 6136 (2020).

113. Ballard, C. et al. Evaluation of the safety, tolerability, and efficacy of pimavanserin versus placebo in patients with Alzheimer's disease psychosis: a phase 2, randomised, placebo-controlled, double-blind study. Lancet Neurol. 17, 213-222 (2018).

114. Sinclair, L. I., Kumar, A., Darreh-Shori, T. \& Love, S Visual hallucinations in Alzheimer's disease do not seem to be associated with chronic hypoperfusion of to visual processing areas V2 and V3 but may be associated with reduced cholinergic input to these areas. Alzheimers Res. Ther. 11, 80 (2019).

115. Watt, J. A. et al. Comparative efficacy of interventions for aggressive and agitated behaviors in dementia: a systematic review and network meta-analysis. Ann. Intern. Med. 171, 633-642 (2019).

116. Ismail, Z. \& Goodarzi, Z. Neuropsychiatric aspects of Alzheimer's disease clinically significant neuropsychiatric symptoms need evidence-based treatment. Practical Neurol. https://practicalneurology.com/articles/ 2019-june/neuropsychiatric-aspects-of-alzheimersdisease (2019).

117. Kales, H. C., Gitlin, L. N., Lyketsos, C. G. \& Detroit Expert Panel on Assessment and Management of Neuropsychiatric Symptoms of Dementia. Management of neuropsychiatric symptoms of dementia in clinical settings: recommendations from a multidisciplinary expert panel. J. Am. Geriatr. Soc. 62, 762-769 (2014).

118. Wolinsky, D., Drake, K. \& Bostwick, J. Diagnosis and management of neuropsychiatric symptoms in Alzheimer's disease. Curr. Psychiatry Rep. 20, 117 (2018).

119. Davies, S. J. et al. Sequential drug treatment algorithm for agitation and aggression in Alzheimer's and mixed dementia. J. Psychopharmacol. 32 509-523 (2018)

120. Ballard, C. et al. in Improving Mental Health and Reducing Antipsychotic Use in People with Dementia in Care Homes: the WHELD Research Programme Including Two RCTS (NIHR Journals Library, 2020)

121. Chen, R. C. et al. Non-pharmacological treatment reducing not only behavioral symptoms, but also psychotic symptoms of older adults with dementia: a prospective cohort study in Taiwan. Geriatr. Gerontol. Int. 14, 440-446 (2014).

122. Gitlin, L. N., Kales, H. C. \& Lyketsos, C. G. Nonpharmacologic management of behavioral symptoms in dementia. JAMA 308, 2020-2029 (2012).

123. Aarsland, D. Impact of dementia-related psychosis on patients and caregivers: the treatment imperative. J. Clin. Psychiatry 81, AD19038BR2C (2020).

124. Ballard, C. Diagnosing dementia-related psychosis: using tools and communicating with patients and caregivers. J. Clin. Psychiatry 82, AD19038BR3C (2020).

125. Schneider, L. S., Dagerman, K. \& Insel, P. S. Efficacy and adverse effects of atypical antipsychotics for dementia: meta-analysis of randomized, placebocontrolled trials. Am. J. Geriatr. Psychiatry 14, 191-210 (2006)

126. Tampi, R. R., Tampi, D. J., Balachandran, S. $\&$ Srinivasan, S. Antipsychotic use in dementia: a systematic review of benefits and risks from meta-analyses. Ther. Adv. Chronic Dis. 7, 229-245 (2016).

127. Ballard, C., Creese, B., Corbett, A. \& Aarsland, D. Atypical antipsychotics for the treatment of behavioral and psychological symptoms in dementia, with a particular focus on longer term outcomes and mortality. Expert Opin. Drug Saf. 10, 35-43 (2011).

128. Maust, D. T. et al. Antipsychotics, other psychotropics, and the risk of death in patients with dementia: number needed to harm. JAMA Psychiatry 72 438-445 (2015)

129. Maher, A. R. et al. Efficacy and comparative effectiveness of atypical antipsychotic medications for off-label uses in adults: a systematic review and meta-analysis. JAMA 306, 1359-1369 (2011).

130. Ballard, C. \& Howard, R. Neuroleptic drugs in dementia: benefits and harm. Nat. Rev. Neurosci. 7, 492-500 (2006).

131. Yunusa, I., Alsumali, A., Garba, A. E., Regestein, Q. R. $\&$ Eguale, T. Assessment of reported comparative effectiveness and safety of atypical antipsychotics in the treatment of behavioral and psychological symptoms of dementia: a network meta-analysis. JAMA Netw. Open. 2, e190828 (2019).

132. Howard, R. et al. Baseline characteristics and treatment-emergent risk factors associated with cerebrovascular event and death with risperidone in dementia patients. Br. J. Psychiatry 209, 378-384 (2016).

133. Reeves, S. et al. Towards safer risperidone prescribing in Alzheimer's disease. Br. J. Psychiatry 218 268-275 (2021).

134. Howard, R. et al. Antipsychotic treatment of very late-onset schizophrenia-like psychosis (ATLAS) a randomised, controlled, double-blind trial. Lancet Psychiatry 5, 553-563 (2018)

135. Malekizadeh, Y. et al. Whole transcriptome in silico screening implicates cardiovascular and infectious disease in the mechanism of action underlying atypical antipsychotic side effects. Alzheimers Dement. 6 , e12078 (2020).

136. Schneider, L. S., Dagerman, K. S. \& Insel, P. Risk of death with atypical antipsychotic drug treatment for dementia: meta-analysis of randomized placebo-controlled trials. JAMA 294, 1934-1943 (2005).

137. Cummings, J. et al. Pimavanserin for patients with Parkinson's disease psychosis: a randomised, placebocontrolled phase 3 trial. Lancet 383, 533-540 (2014).

138. Hacksell, U., Burstein, E. S., McFarland, K., Mills, R. C. $\&$ Williams, H. On the discovery and development of pimavanserin: a novel drug candidate for Parkinson's psychosis. Neurochem. Res. 39, 2008-2017 (2014).

139. Ballard, C., Youakim, J., Coate, B. \& Stankovic, S. Pimavanserin in Alzheimer's disease psychosis: efficacy in patients with more pronounced psychotic symptoms. J. Prev. Alzheimers Dis. 6, 27-33 (2019).

140. ACADIA Pharmaceuticals presents positive top-line results from pivotal phase 3 HARMONY trial of pimavanserin in patients with dementia-related psychosis at 12 th Clinical Trials on Alzheimer's Diseas (CTAD) Meeting. Acadia https://ir.acadia-pharm.com/ news-releases/news-release-details/acadiapharmaceuticals-presents-positive-top-line-results (2020).

141. Ballard, C. G. et al. Long-term evaluation of open-labe pimavanserin safety and tolerability in Parkinson's disease psychosis. Parkinsonism Relat. Disord. 77 100-106 (2020).

142. FDA analysis finds no new or unexpected safety risks associated with Nuplazid (pimavanserin), a medication to treat the hallucinations and delusions of Parkinson's disease psychosis. FDA https://www.fda.gov/drugs/ drug-safety-and-availability/fda-analysis-finds-no-newor-unexpected-safety-risks-associated-nuplazidpimavanserin-medication (2018)

143. Ballard, C. et al. Impact of current antipsychotic medications on comparative mortality and adverse events in people with Parkinson disease psychosis. J. Am. Med. Dir. Assoc. 16, 898.e1-e7 (2015).

144. Porsteinsson, A. P. et al. Effect of citalopram on agitation in Alzheimer disease: the CitAD randomized clinical trial. JAMA 311, 682-691 (2014).

145. Ehrhardt, S. et al. Escitalopram for agitation in Alzheimer's disease (S-CitAD): methods and design of an investigator-initiated, randomized, controlled, multicenter clinical trial. Alzheimers Dement. 15, 1427-1436 (2019).

146. Sheline, Y. I. et al. Effect of escitalopram dose and treatment duration on CSF Abeta levels in healthy older adults: a controlled clinical trial. Neurology 95 , e2658-e2665 (2020).

147. Devanand, D. P. et al. Low dose lithium treatment of behavioral complications in Alzheimer's disease: Lit-AD randomized clinical trial. Am. J. Geriatr. Psychiatry https://doi.org/10.1016/j.jagp.2021.04.014 (2021).

148. Won, E. \& Kim, Y.-K. An oldie but goodie: lithium in the treatment of bipolar disorder through neuroprotective and neurotrophic mechanisms. Int. J. Mol. Sci. 18 2679 (2017)

149. Tan, E. C. et al. Do acetylcholinesterase inhibitors prevent or delay psychotropic prescribing in people with dementia? Analyses of the Swedish Dementia Registry. Am. J. Geriatr. Psychiatry 28, 108-117 (2020).

150. Wang, L. et al. Effects of vitamin D use on outcomes of psychotic symptoms in Alzheimer disease patients. Am. J. Geriatr. Psychiatry 27, 908-917 (2019).

151. Geda, Y. E. et al. Neuropsychiatric symptoms in Alzheimer's disease: past progress and anticipation of the future. Alzheimers Dement. 9, 602-608 (2013)

152. Wise, E. A., Rosenberg, P. B., Lyketsos, C. G. \& Leoutsakos, J.-M. Time course of neuropsychiatric symptoms and cognitive diagnosis in National Alzheimer's Coordinating Centers volunteers. Alzheimers Dement. 11, 333-339 (2019).

153. Hanseeuw, B. J. et al. Association of anxiety with subcortical amyloidosis in cognitively normal older adults. Mol. Psychiatry 25, 2599-2607 (2018).

154. Gatchel, J. R. et al. Longitudinal association of depression symptoms with cognition and cortical amyloid among community-dwelling older adults. JAMA Netw. Open 2, e198964 (2019).

155. Krell-Roesch, J. et al. Cortical beta-amyloid burden, neuropsychiatric symptoms, and cognitive status: the Mayo Clinic Study of Aging. Transl. Psychiatry 9, 123 (2019).

156. Ruthirakuhan, M. T., Ismail, Z., Herrmann, N., Gallagher, D. \& Lanctot, K. Mild behavioral impairment is associated with progression to Alzheimer's disease: results from a clinico-pathological study. Alzheimers Dement. (in the press)

157. Goukasian, N. et al. Association of brain amyloidosis with the incidence and frequency of neuropsychiatric symptoms in ADNI: a multisite observational cohort study. BMJ Open 9, e031947 (2019).

158. Louhija, U.-M., Saarela, T., Juva, K. \& Appelberg, B. Brain atrophy is a frequent finding in elderly patients with first episode psychosis. Int. Psychogeriatr. 29, 1925 (2017).

159. Ismail, Z. et al. Neuropsychiatric symptoms as early manifestations of emergent dementia: provisional diagnostic criteria for mild behavioral impairment. Alzheimers Dement. 12, 195-202 (2016).

160. Taragano, F. E. et al. Risk of conversion to dementia in a mild behavioral impairment group compared to a psychiatric group and to a mild cognitive impairmen group. J. Alzheimers Dis. 62, 227-238 (2018).

161. Ismail, Z et al. The Mild Behavioral Impairment Checklist (MBI-C): a rating scale for neuropsychiatric symptoms in pre-dementia populations. J. Alzheimers Dis. 56, 929-938 (2017).

162. Creese, B. et al. Mild behavioral impairment as a marker of cognitive decline in cognitively normal older adults. Am. J. Geriatr. Psychiatry 27, 823-834 (2019).

163. Creese, B. et al. Profile of mild behavioral impairment and factor structure of the mild behavioral impairment checklist in cognitively normal older adults. Int. Psychogeriatr. 32, 705-717 (2020).

164. Hu, S., Patten, S. B., Fick, G. Smith, E. E. \& Ismail, Z Validation of the mild behavioral impairment checklist (MBI-C) in a clinic-based sample. Alzheimers Dement. 15, P365 (2019)

165. Mallo, S. C. et al. Assessing mild behavioral impairment with the mild behavioral impairment-checklist in people with mild cognitive impairment. J. Alzheimers Dis. 66, 83-95 (2018).

166. Mallo, S. C. et al. Assessing mild behavioral impairment with the mild behavioral impairment checklist in people with subjective cognitive decline. Int. Psychogeriatr. 31, 231-239 (2019)

167. Matsuoka, T., Ismail, Z. \& Narumoto, J. Prevalence of mild behavioral impairment and risk of dementia in a psychiatric outpatient clinic. J. Alzheimers Dis. 70 505-513 (2019).

168. Kassam, F. et al. Cognitive profile of mild behavioral impairment (MBI) in brain health registry participants: developing topics. Alzheimers Dement. 16, e047673 (2020).

169. Ismail, Z. et al. Prevalence of depression in patients with mild cognitive impairment: a systematic review and meta-analysis. JAMA Psychiatry 74, 58-67 (2017).

170. Ismail, Z. et al. Mild behavioral impairment and subjective cognitive decline predict cognitive and functional decline. J. Alzheimers Dis. 80 459-469 (2021).

171. Nathan, S., Gill, S. \& Ismail, Z. in Alzheimer's Association International Conference (ALZ, 2020).

172. Lussier, F. Z. et al. Mild behavioral impairment is associated with $\beta$-amyloid but not tau or neurodegeneration in cognitively intact elderly individuals. Alzheimers Dement. 16, 192-199 (2020).

173. Miao, R. et al. Plasma $\beta$-amyloid in mild behavioural impairment-neuropsychiatric symptoms on the Alzheimer's continuum. J. Geriatr. Psychiatry Neurol. https://doi.org/10.1177/08919887211016068 (2021).

174. Lussier, F. et al. Mild behavioral impairment is associated with beta-amyloid and tau across the alzheimer's disease spectrum. J. Cereb. Blood Flow. Metab. 39, 158-159 (2019).

175. Johansson, M. et al. Mild behavioral impairment and its relation to tau pathology in preclinical Alzheimer's disease. Transl. Psychiatry 11, 76 (2021).

176. Gill, S. et al. Diffusion tensor imaging in pre-dementia risk states: white matter atrophy findings in mild 
behavioral impairment (P5. 1-025). Neurology 92, P5.1-025 (2019).

177. Yoon, E. et al. Mild behavioral impairment is linked to worse cognition and brain atrophy in Parkinson's disease. Neurology 93, e766-e777 (2019).

178. Naude, J. et al. Plasma neurofilament Light: a marker of cognitive decline in mild behavioural impairment. J. Alzheimers Dis. 76, 1017-1027 (2020).

179. Andrews, S. J., Ismail, Z., Anstey, K. J. \& Mortby, M. Association of Alzheimer's genetic loci with mild behavioral impairment. Am. J. Med. Genet. B Neuropsychiatr. Genet. 177, 727-735 (2018)

180. Creese, B. et al. Genetic risk for Alzheimer's disease, cognition, and mild behavioral impairment in healthy older adults. Alzheimers Dement. 13, e12164 (2021).

181. Wolfova, K. et al. Sex differences in the association of mild behavioral impairment with cognitive aging. Preprint at medRxiv https://doi.org/10.1101/ 2021.05.20.21257514 (2021).

182. Mortby, M. E. et al. Dementia clinical trial implications of mild behavioral impairment. Int. Psychogeriatr. 30, 171-175 (2018).
183. Gerlach, L. B. et al. Trends in antipsychotic and mood stabilizer prescribing in long-term care in the US:

2011-2014. J. Am. Med. Dir. Assoc. 21, 1629-1635.e8 (2020).

Author contributions

The authors contributed equally to all aspects of the article.

\section{Competing interests}

Z.I. has received research funding from Canadian Institutes of Health Research and Brain Canada, and has received persona fees from Lundbeck and Otsuka not related to the submitted work. His institution has received fees from Acadia, Biogen, Roche and Sunovion. D.A. has received research suppor and/or honoraria from AstraZeneca, H. Lundbeck, Novartis Pharmaceuticals, and GE Health and serves as a paid consultant for H. Lundbeck, Eisai and Axovant. C.G.L. has received grant support (research or Continuing Medical Education) from the National Institute of Mental Health, the National Institute on Aging, the Associated Jewish Federation of Baltimore, the Weinberg Foundation, Forest, GlaxoSmithKline, Eisai, Pfizer, AstraZeneca, Lilly, Ortho-McNeil, Bristol Myers Squibb, Novartis, the National Football League (NFL), Elan, Functional
Neuromodulation and Bright Focus Foundation; received payment as a consultant or adviser from AstraZeneca, GlaxoSmithKline, Eisai, Novartis, Forest, Supernus, Adlyfe, Takeda, Wyeth, Lundbeck, Merz, Lilly, Pfizer, Genentech, Elan, NFL Players Association, NFL Benefits Office, Avanir, Zinfandel, BMS, AbbVie, Janssen, Orion, Otsuka, Servier and Astellas, and received honoraria or travel support from Pfizer, Forest, GlaxoSmithKline, and Health Monitor. Clive Ballard has received grants and personal fees from Acadia and Lundbeck, and personal fees from Heptares, Roche, Lilly, Otsuka, Orion, GlaxoSmithKline and Pfizer. B.C., H.C.K. and R.A.S. declare no competing interests.

\section{Peer review information}

Nature Reviews Neurology thanks S. Reeves and the other, anonymous, reviewer(s) for their contribution to the peer review of this work.

\section{Publisher's note}

Springer Nature remains neutral with regard to jurisdictional claims in published maps and institutional affiliations.

(c) Springer Nature Limited 2022 